\title{
THE MINIMUM RANK PROBLEM OVER FINITE FIELDS*
}

\author{
JASON GROUT ${ }^{\dagger}$
}

\begin{abstract}
The structure of all graphs having minimum rank at most $k$ over a finite field with $q$ elements is characterized for any possible $k$ and $q$. A strong connection between this characterization and polarities of projective geometries is explained. Using this connection, a few results in the minimum rank problem are derived by applying some known results from projective geometry.
\end{abstract}

Key words. Minimum rank, Symmetric matrix, Finite field, Projective geometry, Polarity graph, Bilinear symmetric form

AMS subject classifications. 05C50, 05C75, 15A03, 05B25, 51E20.

1. Introduction. Given a field $F$ and a simple undirected graph $G$ on $n$ vertices (i.e., an undirected graph without loops or multiple edges), let $S(F, G)$ be the set of symmetric $n \times n$ matrices $A$ with entries in $F$ satisfying $a_{i j} \neq 0, i \neq j$, if and only if $i j$ is an edge in $G$. There is no restriction on the diagonal entries of the matrices in $S(F, G)$. Let

$$
\operatorname{mr}(F, G)=\min \{\operatorname{rank} A \mid A \in S(F, G)\} .
$$

Let $\mathcal{G}_{k}(F)=\{G \mid \operatorname{mr}(F, G) \leq k\}$, the set of simple graphs with minimum rank at most $k$.

The problem of finding $\operatorname{mr}(F, G)$ and describing $\mathcal{G}_{k}(F)$ has recently attracted considerable attention, particularly for the case in which $F=\mathbb{R}$ (see $[29,17,26,25$, $27,13,33,5,9,22,2,11,6,7,10,18,4])$. The minimum rank problem over $\mathbb{R}$ is a sub-problem of a much more general problem, the inverse eigenvalue problem for symmetric matrices: given a family of real numbers, find every symmetric matrix that has the family as its eigenvalues. More particularly, the minimum rank problem is a sub-problem of the inverse eigenvalue problem for graphs, which fixes a zero/nonzero pattern for the symmetric matrices considered in the inverse eigenvalue problem. The minimum rank problem can also be thought of in this way: given a fixed pattern of off-diagonal zeros, what is the smallest rank that a symmetric matrix having that pattern can achieve?

${ }^{*}$ Received by the editors January 17, 2008. Accepted for publication July 13, 2010. Handling Editor: Robert Guralnick.

$\dagger$ Department of Mathematics and Computer Science, Drake University, 2507 University Avenue, Des Moines, IA 50311, USA (jason.grout@drake.edu). 


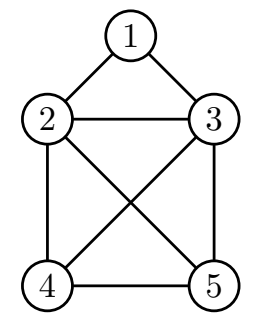

Fig. 1.1: A labeled full house graph

Up to the addition of isolated vertices, it is easy to see that $\mathcal{G}_{1}(F)=\left\{K_{n} \mid\right.$ $n \in \mathbb{N}\}$ for any field $F$. In [9] and [10], $\mathcal{G}_{2}(F)$ was characterized for any field $F$ both in terms of forbidden subgraphs and in terms of the structure of the graph complements. The forbidden subgraph characterizations in these papers used ten or fewer graphs for each value of $k$. Restricting our focus to finite fields, let $\mathbb{F}_{q}$ denote the finite field with $q$ elements. Ding and Kotlov [18] independently used structures similar to the structures that we use in this paper to obtain some special cases of some structural results in this paper, as well as an upper bound for the sizes of minimal forbidden subgraphs characterizing $\mathcal{G}_{k}\left(\mathbb{F}_{q}\right)$ for any $k$ and any $q$. The latter result implies that there are a finite number of forbidden subgraphs characterizing $\mathcal{G}_{k}\left(\mathbb{F}_{q}\right)$. For example, in [8], $\mathcal{G}_{3}\left(\mathbb{F}_{2}\right)$ was characterized by 62 forbidden subgraphs. This characterization and further computations confirm our intuition that the forbidden subgraph characterizations of $\mathcal{G}_{k}\left(\mathbb{F}_{q}\right)$ quickly become complicated as $k$ increases.

In this paper, we will characterize the structure of graphs in $\mathcal{G}_{k}\left(\mathbb{F}_{q}\right)$ for any $k$ and any $q$. The characterization is simply stated and has a very strong connection to projective geometry over finite fields. At the end of the paper, we will list a few of the ramifications of this connection to projective geometry.

We adopt the following notation dealing with fields, vector spaces, and matrices. Given a field $F$, the group of nonzero elements under multiplication is denoted $F^{\times}$and the vector space of dimension $k$ over $F$ is denoted $F^{k}$. Given a matrix $M$, the principal submatrix lying in the rows and columns $x_{1}, x_{2}, \ldots, x_{m}$ is denoted $M\left[x_{1}, x_{2}, \ldots, x_{m}\right]$.

To illustrate the field-dependence of minimum rank, we recall from [10] the full house graph in Figure 1.1 (there called $\left(P_{3} \cup 2 K_{1}\right)^{c}$ ), which is the only graph on 5 or fewer vertices for which the minimum rank is field-dependent. 
If $F \neq \mathbb{F}_{2}$, there are elements $a, b \neq 0$ in $F$ such that $a+b \neq 0$. Then

$$
\left[\begin{array}{ccccc}
a & a & a & 0 & 0 \\
a & a+b & a+b & b & b \\
a & a+b & a+b & b & b \\
0 & b & b & b & b \\
0 & b & b & b & b
\end{array}\right] \in S(F, \text { full house })
$$

which shows that $\operatorname{mr}(F$, full house $)=2$. The case $F=\mathbb{F}_{2}$ gives a different result. Let $A$ be any matrix in $S\left(\mathbb{F}_{2}\right.$, full house). Then for some $d_{1}, d_{2}, \ldots, d_{5} \in \mathbb{F}_{2}$,

$A=\left[\begin{array}{ccccc}d_{1} & 1 & 1 & 0 & 0 \\ 1 & d_{2} & 1 & 1 & 1 \\ 1 & 1 & d_{3} & 1 & 1 \\ 0 & 1 & 1 & d_{4} & 1 \\ 0 & 1 & 1 & 1 & d_{5}\end{array}\right]$ and $\operatorname{det}(A[\{1,2,5\},\{1,3,4\}])=\left|\begin{array}{ccc}d_{1} & 1 & 0 \\ 1 & 1 & 1 \\ 0 & 1 & 1\end{array}\right|=1$,

where $A[\{1,2,5\},\{1,3,4\}]$ is the submatrix of $A$ lying in rows $\{1,2,5\}$ and columns $\{1,3,4\}$. Therefore $\operatorname{mr}\left(\mathbb{F}_{2}\right.$, full house $) \geq 3$. Setting each $d_{i}$ to 1 verifies the statement that $\operatorname{mr}\left(\mathbb{F}_{2}\right.$, full house $)=3$.

In spite of this dependence on the field, there are a number of results about minimum rank that are field independent. For example, the minimum rank of a tree is field independent (see any of [3], [31], or [14]). Many of the forbidden subgraphs classifying $\mathcal{G}_{3}\left(\mathbb{F}_{2}\right)$ that are found in [8] are also forbidden subgraphs for $\mathcal{G}_{3}(F)$ for any field $F$. These results and others demonstrate that results obtained over finite fields can provide important insights for other fields.

The presentation of material in this paper is oriented towards a reader that is familiar with concepts from linear algebra and graph theory. In the rest of this section, we will review some of our conventions in terminology from graph theory.

In this paper, graphs are undirected, may have loops, but will not have multiple edges between vertices. To simplify our drawings, a vertex with a loop (a looped vertex) will be filled (black) and a vertex without a loop (a nonlooped vertex) will be empty (white). A simple graph is a graph without loops. Let $G$ be a graph with some loops and $\hat{G}$ be the simple version of $G$ obtained by deleting all loops. We say that a matrix in $S(F, \hat{G})$ corresponds to the simple graph $\hat{G}$. A matrix $A \in S(F, \hat{G})$ corresponds to $G$ if $a_{i i}$ is nonzero exactly when the vertex $i$ has a loop in $G$. Note that if a matrix corresponds to a looped graph, then it also corresponds to the simple version of the graph.

We recall some notation from graph theory.

Definition 1.1. Given two graphs $G$ and $H$ with disjoint vertex sets $V(G)$ and $V(H)$ and edge sets $E(G)$ and $E(H)$, the union of $G$ and $H$, denoted $G \cup H$, has 


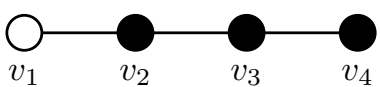

(a) $G$

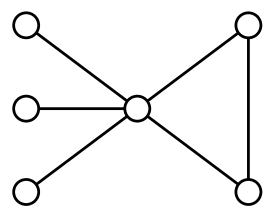

(b) $H$, a blowup of $G$

Fig. 1.2: Graphs in Example 1.5

vertices $V(G) \cup V(H)$ and edges $E(G) \cup E(H)$. The join of $G$ and $H$, denoted $G \vee H$, has vertices $V(G) \cup V(H)$ and edges $E(G) \cup E(H) \cup\{u v \mid u \in V(G), v \in V(H)\}$. The complement of the graph $G$, denoted $G^{c}$, has vertices $V(G)$ and edges $\{u v \mid u, v \in$ $V(G), u v \notin E(G)\}$. Note that a vertex is looped in $G$ if and only if it is nonlooped in $G^{c}$.

Definition 1.2. The simple complete graph on $n$ vertices will be denoted by $K_{n}$ and has vertices $\{1,2, \ldots, n\}$ and edges $\left\{x y \mid x, y \in V\left(K_{n}\right), x \neq y\right\}$. The simple complete multipartite graph $K_{s_{1}, s_{2}, \ldots, s_{m}}$ is defined as $K_{s_{1}}^{c} \vee K_{s_{2}}^{c} \vee \cdots \vee K_{s_{m}}^{c}$.

Definition 1.3. Two vertices in a graph are adjacent if an edge connects them. A clique in a graph is a set of pairwise adjacent vertices. An independent set in a graph is a set of pairwise nonadjacent vertices.

The next definition extends a standard definition introduced in [28] and is used in random graph theory in connection with the regularity lemma.

Definition 1.4. A blowup of a graph $G$ with vertices $\left\{v_{1}, v_{2}, \ldots, v_{n}\right\}$ is a new simple graph $H$ constructed by replacing each nonlooped vertex $v_{i}$ in $G$ with a (possibly empty) independent set $V_{i}$, each looped vertex $v_{i}$ with a (possibly empty) clique $V_{i}$, and each edge $v_{i} v_{j}$ in $G(i \neq j)$ with the edges $\left\{x y \mid x \in V_{i}, y \in V_{j}\right\}$ in $H$.

EXAmPLe 1.5. Let $G$ be the graph labeled in Figure 1.2(a).

Let $\left|V_{1}\right|=3,\left|V_{2}\right|=1,\left|V_{3}\right|=2$, and $\left|V_{4}\right|=0$. Then we obtain the simple blowup graph $H$ in Figure 1.2(b). It is useful to see how matrices corresponding to a graph and a blowup of the graph are related. Over $\mathbb{F}_{3}$, let

$$
M=\left[\begin{array}{c|c|c|c}
0 & 2 & 0 & 0 \\
\hline 2 & 1 & 1 & 0 \\
\hline 0 & 1 & 1 & 1 \\
\hline 0 & 0 & 1 & 1
\end{array}\right] \quad \text { and } \quad N=\left[\begin{array}{ccc|c|cc}
0 & 0 & 0 & 1 & 0 & 0 \\
0 & 0 & 0 & 2 & 0 & 0 \\
0 & 0 & 0 & 1 & 0 & 0 \\
\hline 1 & 2 & 1 & 0 & 1 & 1 \\
\hline 0 & 0 & 0 & 1 & 0 & 1 \\
0 & 0 & 0 & 1 & 1 & 2
\end{array}\right] \text {. }
$$




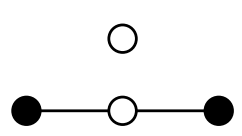

(a)

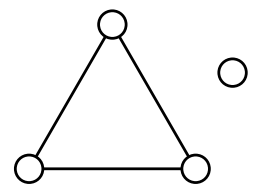

(b)

Fig. 2.1: Graphs in Theorem 2.2

Then $M$ is an example of a matrix corresponding to $G$ and $N$ is an example of a matrix corresponding to $H$. Note that, for example, the entry $m_{11}$ was replaced with a $3 \times 3$ zero block in $N$, the entry $m_{12}$ was replaced with a $3 \times 1$ nonzero block in $N$, the entries in the last row and column of $M$ were replaced with empty blocks (i.e., erased), and the diagonal entries of $N$ were changed to whatever was desired. These substitutions of block matrices correspond to the vertex substitutions used to construct $H$.

We will introduce our method by presenting a proof of a special case of a characterization theorem from [10] which characterizes $\mathcal{G}_{2}\left(\mathbb{F}_{2}\right)$. We will then generalize this proof into a characterization of all simple graphs in $\mathcal{G}_{k}\left(\mathbb{F}_{q}\right)$ for any $k$ and $q$. After giving examples for some specific $k$ and $q$, we will describe the strong connection to projective geometry and list some consequences of this connection.

2. A new approach to a recent result. We will introduce our method by giving a proof of a special case of Theorems 5 and 6 of [10].

TheOREM 2.1 ([10]). Let $G$ be a simple graph on $n$ vertices. Then $\operatorname{mr}\left(\mathbb{F}_{2}, G\right) \leq 2$ if and only if the simple version of $G^{c}$ is either of the form

$$
\left(K_{s_{1}} \cup K_{p_{1}, q_{1}}\right) \vee K_{r}
$$

for some appropriate nonnegative integers $s_{1}, p_{1}, q_{1}$, and $r$, or of the form

$$
\left(K_{s_{1}} \cup K_{s_{2}} \cup K_{s_{3}}\right) \vee K_{r}
$$

for some appropriate nonnegative integers $s_{1}, s_{2}, s_{3}$, and $r$.

We first rephrase Theorem 2.1 using blowup graph terminology.

TheOREm 2.2 ([10]). Let $G$ be a simple graph on $n$ vertices. Then $\operatorname{mr}\left(\mathbb{F}_{2}, G\right) \leq 2$ (i.e., $G \in \mathcal{G}_{2}\left(\mathbb{F}_{2}\right)$ ) if and only if $G$ is a blowup of either of the graphs in Figure 2.1.

In the proof of this result, we will need the following lemma and corollary, which hold in any field. We will then give a proof of Theorem 2.2. 
Lemma 2.3 ([15, Theorem 8.9.1]). Let $A$ be an $n \times n$ symmetric matrix of rank $k$. Then there is an invertible principal $k \times k$ submatrix $B$ of $A$ and $a k \times n$ matrix $U$ such that

$$
A=U^{t} B U
$$

Corollary 2.4. Let $A$ be an $n \times n$ symmetric matrix. Then $\operatorname{rank} A \leq k$ if and only if there is some invertible $k \times k$ matrix $B$ and $k \times n$ matrix $U$ such that $A=U^{t} B U$.

Proof. Let $A$ have rank $r \leq k$. Then by Lemma 2.3, there is an invertible $r \times r$ matrix $B_{1}$ and an $r \times n$ matrix $U_{1}$ such that $A=U_{1}^{t} B_{1} U_{1}$. Let $B_{2}=\left[\begin{array}{cc}B_{1} & O \\ O & I_{k-r}\end{array}\right]$ and $U_{2}=\left[\begin{array}{c}U_{1} \\ O\end{array}\right]$ (where $O$ represents a zero matrix of the appropriate size). Then $A=U_{2}^{t} B_{2} U_{2}$. The reverse implication follows from the $\operatorname{rank}$ inequality $\operatorname{rank}\left(U^{t} B U\right) \leq$ rank $B$. $\square$

Recall that two square matrices $A$ and $B$ are congruent if there exists some invertible matrix $C$ such that $A=C^{t} B C$. It is straightforward to show that congruence is an equivalence relation. Let $\mathcal{B}$ consist of one representative from each congruence equivalence class of invertible symmetric $k \times k$ matrices. By Corollary 2.4, if $A$ is a symmetric $n \times n$ matrix with $\operatorname{rank} A \leq k$, then $A \in\left\{U^{t} B U \mid B \in \mathcal{B}, U\right.$ a $k \times n$ matrix $\}$.

We now proceed with the proof of Theorem 2.2.

Proof. [Proof of Theorem 2.2] First, we compute a suitable $\mathcal{B}$, a set of representatives from the congruence classes of invertible symmetric $2 \times 2$ matrices over $\mathbb{F}_{2}$. If an invertible symmetric $2 \times 2$ matrix $B$ over $\mathbb{F}_{2}$ has a nonzero diagonal entry, then $B=\left[\begin{array}{ll}1 & 1 \\ 1 & 0\end{array}\right], B=\left[\begin{array}{ll}0 & 1 \\ 1 & 1\end{array}\right]$, or $B=I_{2}$. In any of these three cases, $B^{t} B B=I_{2}$, so $B$ is congruent to the identity matrix $I_{2}$. If an invertible symmetric $2 \times 2$ matrix $B$ over $\mathbb{F}_{2}$ has all zeros on the diagonal, then the off-diagonal entries must be nonzero, so $B=\left[\begin{array}{ll}0 & 1 \\ 1 & 0\end{array}\right]$. In this case,

$$
\left[\begin{array}{ll}
a & c \\
b & d
\end{array}\right]\left[\begin{array}{ll}
0 & 1 \\
1 & 0
\end{array}\right]\left[\begin{array}{ll}
a & b \\
c & d
\end{array}\right]=\left[\begin{array}{ll}
a c+a c & a d+b c \\
a d+b c & b d+b d
\end{array}\right]=\left[\begin{array}{cc}
0 & a d+b c \\
a d+b c & 0
\end{array}\right]
$$

so any matrix congruent to $B$ will have a zero diagonal. Therefore, a suitable $\mathcal{B}$ is

$$
\mathcal{B}=\left\{I_{2},\left[\begin{array}{ll}
0 & 1 \\
1 & 0
\end{array}\right]\right\}
$$


Because $U$ is a matrix with entries in $\mathbb{F}_{2}$, the columns of $U$ are members of the finite set

$$
\left\{\left[\begin{array}{l}
1 \\
0
\end{array}\right],\left[\begin{array}{l}
0 \\
1
\end{array}\right],\left[\begin{array}{l}
1 \\
1
\end{array}\right],\left[\begin{array}{l}
0 \\
0
\end{array}\right]\right\} .
$$

Let $A$ be a symmetric $k \times k$ matrix. For any $n \times n$ permutation matrix $P$, the graphs of $A$ and $P^{t} A P$ are isomorphic. Therefore we may assume that identical columns of $U$ are contiguous and write $U=\left[\begin{array}{llll}E_{1} & E_{2} & J & O\end{array}\right]$ where $E_{1}$ is $2 \times p$ matrix with each column equal to $\left[\begin{array}{l}1 \\ 0\end{array}\right], E_{2}$ is $2 \times q$ matrix with each column equal to $\left[\begin{array}{l}0 \\ 1\end{array}\right], J$ is a $2 \times r$ matrix with each entry equal to 1 , and $O$ is a $2 \times t$ zero matrix. Then either

$$
A=\left[\begin{array}{c}
E_{1}^{\mathrm{T}} \\
E_{2}^{\mathrm{T}} \\
J^{\mathrm{T}} \\
O^{\mathrm{T}}
\end{array}\right]\left[\begin{array}{llll}
E_{1} & E_{2} & J & O
\end{array}\right]=\left[\begin{array}{cccc}
J_{p} & O & J_{p, r} & O \\
O & J_{q} & J_{q, r} & O \\
J_{r, p} & J_{r, q} & O_{r} & O \\
O & O & O & O_{t}
\end{array}\right]
$$

or else

$$
A=\left[\begin{array}{c}
E_{1}^{\mathrm{T}} \\
E_{2}^{\mathrm{T}} \\
J^{\mathrm{T}} \\
O^{\mathrm{T}}
\end{array}\right]\left[\begin{array}{ll}
0 & 1 \\
1 & 0
\end{array}\right]\left[\begin{array}{llll}
E_{1} & E_{2} & J & O
\end{array}\right]=\left[\begin{array}{cccc}
O_{p} & J_{p, q} & J_{p, r} & O \\
J_{q, p} & O_{q} & J_{q, r} & O \\
J_{r, p} & J_{r, q} & O_{r} & O \\
O & O & O & O_{t}
\end{array}\right],
$$

where $J$ is an all-ones matrix, $O$ is a zero matrix, and subscripts of $J$ and $O$ denote the dimensions of the matrix.

Any simple graph corresponding to the first matrix is a blowup of the graph in Figure 2.1(a), while any simple graph corresponding to the second matrix is a blowup of the graph in Figure 2.1(b). Thus we have established Theorem 2.2.

ObSERVATION 2.5. Note that every block in the above matrices is either a $O$ matrix or a $J$ matrix. Consequently, we could have obtained the zero/nonzero form of the matrices with rank at most 2 by only considering $U=\left[\begin{array}{llll}1 & 0 & 1 & 0 \\ 0 & 1 & 1 & 0\end{array}\right]$ and computing

$$
A=U^{t} U=\left[\begin{array}{llll}
1 & 0 & 1 & 0 \\
0 & 1 & 1 & 0 \\
1 & 1 & 0 & 0 \\
0 & 0 & 0 & 0
\end{array}\right]
$$

and

$$
A=U^{t} B_{2} U=\left[\begin{array}{ll}
1 & 0 \\
0 & 1 \\
1 & 1 \\
0 & 0
\end{array}\right]\left[\begin{array}{llll}
0 & 1 & 1 & 0 \\
1 & 0 & 1 & 0
\end{array}\right]=\left[\begin{array}{llll}
0 & 1 & 1 & 0 \\
1 & 0 & 1 & 0 \\
1 & 1 & 0 & 0 \\
0 & 0 & 0 & 0
\end{array}\right]
$$


The nonzero diagonal entries correspond to loops in our graphs. This simplified procedure again yields the graphs in Figure 2.1.

In the proof of Theorem 2.2, we noted that any $U$ could be written in a standard form. In Observation 2.5, we saw how the standard form of $U$ could be simplified to take advantage of the theorem being about blowup graphs. We will now discuss the reasoning behind these constructions and show that an analogous standard form of $U$ exists for any finite field and any $k$.

Because we construct the graphs using representatives of congruence classes, it is important for any simplified $U$ to have the property that if $B$ and $\hat{B}$ are congruent, then $U^{t} B U$ and $U^{t} \hat{B} U$ correspond to isomorphic graphs. The following lemma shows that if we take a matrix $U$ where the columns consist of all vectors in $\mathbb{F}_{q}^{k}$, like in Observation 2.5, and if $B$ and $\hat{B}$ are congruent, then $U^{t} B U$ and $U^{t} \hat{B} U$ correspond to isomorphic graphs.

Lemma 2.6. Let $U$ be the matrix with columns $\left\{v \mid v \in \mathbb{F}_{q}^{k}\right\}$. Let $B$ and $C$ be invertible $k \times k$ matrices with $B$ symmetric. Then the graphs corresponding to $U^{t} B U$ and $U^{t}\left(C^{t} B C\right) U$ are isomorphic.

Proof. Since every vector in $\mathbb{F}_{q}^{k}$ appears as a column of $U$ and the mapping $x \mapsto C x$ is one-to-one, $C U$ is just a column permutation of $U$. This permutation induces a relabeling of the graph $U^{t} B U$ to give the graph of $(C U)^{t} B(C U)=U^{t}\left(C^{t} B C\right) U$.

Though this invariance property with respect to congruent matrices does not hold for an arbitrary $U$, there is another smaller $U$ which does have the same property. We first need some preliminary material. Then we will introduce this new $U$ in Lemma 2.9 .

Definition 2.7. Let $F$ be a field. Two nonzero vectors $v_{1}, v_{2} \in F^{k}$ are projectively equivalent if there exists some nonzero $c \in F$ such that $v_{1}=c v_{2}$.

It is easy to check that projective equivalence is in fact an equivalence relation on the vectors in $V$.

We pause to note that replacing a column of $U$ with a projectively equivalent column does not affect the graph corresponding to $U^{t} B U$. To see this, let $U=$ $\left[\begin{array}{llll}u_{1} & u_{2} & \cdots & u_{n}\end{array}\right]$ and let $i \in\{1,2, \ldots, n\}$. Let $\hat{U}$ be the matrix obtained from $U$ by replacing the column $u_{i}$ with $c u_{i}$ for some nonzero $c \in F$. Then the $i, j$ entry of $\hat{U}^{t} B \hat{U},\left(c u_{i}\right)^{t} B u_{j}$ if $i \neq j$ or $\left(c u_{i}\right)^{t} B\left(c u_{i}\right)$ if $i=j$, is zero if and only if the $i, j$ entry of $U^{t} B U, u_{i}^{t} B u_{j}$, is zero. Thus the graphs associated with $U^{t} B U$ and $\hat{U}^{t} B \hat{U}$ are equal.

Lemma 2.8. Let $F$ be any field, let $x \in F^{k}$, let $\bar{x}$ denote the projective equivalence class of $x$, and let $P=\cup_{x \in F^{k}-\overrightarrow{0}}\{\bar{x}\}$, the set of projective equivalence classes in $F^{k}$. Let $C$ be an invertible matrix. Then the map $f: P \rightarrow P$ defined by $f: \bar{x} \mapsto \overline{C x}$ is a 
bijection.

Proof. The function $f$ is well-defined since if $C x=y$, then for any nonzero $k \in F$, $\overline{C(k x)}=\overline{k C x}=\overline{k y}=\bar{y}$. If $\overline{C x_{1}}=\overline{C x_{2}}$, then for some nonzero $k \in F, k C x_{1}=C x_{2}$, which implies $C\left(k x_{1}-x_{2}\right)=0$, giving $k x_{1}=x_{2}$ since $C$ is invertible. Therefore $\overline{x_{1}}=\overline{x_{2}}$ and $f$ is injective. Surjectivity of $f$ also follows from the hypothesis that $C$ is invertible.

LEMMA 2.9. Let $\overline{x_{1}}, \overline{x_{2}}, \ldots, \overline{x_{m}}$ be the projective equivalence classes of $\mathbb{F}_{q}^{k}-\overrightarrow{0}$, with each $x_{i}$ as a chosen representative from its class. Let $U=\left[\begin{array}{llll}x_{1} & x_{2} & \cdots & x_{m}\end{array}\right]$, the matrix with column vectors $x_{1}, x_{2}, \ldots, x_{m}$. Let $B$ and $C$ be invertible $k \times k$ matrices with $B$ symmetric. Then the graphs corresponding to $U^{t} B U$ and $U^{t}\left(C^{t} B C\right) U$ are isomorphic.

Proof. Let $T=C U$. Denote the $i$ th column of $U$ by $u_{i}$ and the $i$ th column of $T$ by $t_{i}$. By Lemma 2.8, the sequence of projective equivalence classes $\overline{t_{1}}, \overline{t_{2}}, \ldots, \overline{t_{n}}$ is just a permutation of the sequence $\overline{u_{1}}, \overline{u_{2}}, \ldots, \overline{u_{n}}$. Form the matrix $S$ in which the $i$ th column, $s_{i}$, is $u_{j}$ if $\overline{t_{i}}=\overline{u_{j}}$, so that $S$ is a column permutation of $U$ and $\overline{s_{i}}=\overline{t_{i}}$. Then the graph corresponding to $U^{t}\left(C^{t} B C\right) U=(C U)^{t} B(C U)=T^{t} B T$ is isomorphic to the graph corresponding to $S^{t} B S$ by the reasoning preceding Lemma 2.8, which is in turn just a relabeling of the graph corresponding to $U^{t} B U$.

We now find a standard form for any matrix $U$, as in our proof of Theorem 2.2. Let $U$ be a $k \times n$ matrix over $\mathbb{F}_{q}$ and let $B$ be an invertible symmetric $k \times k$ matrix over $\mathbb{F}_{q}$. Let $\overline{x_{1}}, \overline{x_{2}}, \ldots, \overline{x_{m}}$ be the projective equivalence classes of $\mathbb{F}_{q}^{k}-\overrightarrow{0}$, with each $x_{i}$ as a chosen representative from its class. For each nonzero column $u_{i}$ of $U$, replace $u_{i}$ with the chosen representative of $\overline{u_{i}}$. Then permute the columns of $U$ so that the matrix is of the form $\hat{U}=\left[\begin{array}{lllll}X_{1} & X_{2} & \cdots & X_{m} O\end{array}\right]$, where each $X_{i}$ is a block matrix of columns equal to $x_{i}$ and $O$ is a zero block matrix. Note that some of these blocks may be empty. Let $G$ be the simple graph corresponding to $U^{t} B U$ and let $\hat{G}$ be the simple graph corresponding to $\hat{U}^{t} B \hat{U}$. From our results above, $G$ is isomorphic to $\hat{G}$.

As illustrated in Observation 2.5, we can obtain the zero/nonzero structure of the block matrix $\hat{U}^{t} B \hat{U}$ by simply deleting all duplicate columns of $\hat{U}$. Deleting these duplicate columns of $\hat{U}$ leaves a matrix that can be obtained from $\tilde{U}=\left[\begin{array}{llll}x_{1} & x_{2} & \cdots & x_{m}\end{array}\right]$ by deleting the columns of $\tilde{U}$ corresponding to empty blocks of $\hat{U}$. Let $\tilde{G}$ be the (looped) graph corresponding to $\tilde{U}^{t} B \tilde{U}$. Then $\hat{G}$ is a blowup of $\tilde{G}$, which implies that $G$ is a blowup of $\tilde{G}$.

Furthermore, let $\mathcal{B}$ be a set consisting of one representative from each congruence class of invertible symmetric $k \times k$ matrices and let $\hat{B}$ be the representative that is congruent to $B$. Then from Lemma 2.9, the graphs corresponding to $\tilde{U}^{t} B \tilde{U}$ and $\tilde{U}^{t} \hat{B} \tilde{U}$ are isomorphic. 
There is another simplification we can make. Notice that both graphs displayed in Theorem 2.2 have an isolated nonlooped vertex. This vertex came from the zero column vectors in $U$ and corresponds to the fact that adding any number of isolated vertices to a graph does not change its minimum rank. In any theorem like Theorem 2.2, each graph from which we construct blowups will always have this isolated nonlooped vertex and so will be of the form $G \cup K_{1}$. Note that in constructing such a graph $G$, it is enough to assume that $\tilde{U}$ in the above paragraphs does not have a zero column vector.

DEFINITION 2.10. Let $\overline{x_{1}}, \overline{x_{2}}, \ldots, \overline{x_{m}}$ be the projective equivalence classes of $\mathbb{F}_{q}^{k}-\overrightarrow{0}$, with each $x_{i}$ as a chosen representative from its class. Let $\mathcal{B}$ be a set consisting of one representative from each congruence class of invertible symmetric $k \times k$ matrices. Let $U=\left[\begin{array}{llll}x_{1} & x_{2} & \cdots & x_{m}\end{array}\right]$, the matrix with column vectors $x_{1}, x_{2}, \ldots, x_{m}$. We define the set of graphs $\mathfrak{g}_{k}\left(\mathbb{F}_{q}\right)$ as the set of graphs corresponding to the matrices in $\left\{U^{t} B U\right.$ | $B \in \mathcal{B}\}$.

We now have the following result (recall that $K_{1}$ has no loop).

TheOREM 2.11. A simple graph $G$ is in $\mathcal{G}_{k}\left(\mathbb{F}_{q}\right)$ if and only if $G$ is a blowup of some graph in $\left\{H \cup K_{1} \mid H \in \mathfrak{g}_{k}\left(\mathbb{F}_{q}\right)\right\}$.

Proof. Let $G$ be a simple graph in $\mathcal{G}_{k}\left(\mathbb{F}_{q}\right)$. Let $A \in S\left(\mathbb{F}_{q}, G\right)$ be a matrix with $\operatorname{rank} A \leq k$. Then $A=U^{t} B U$ for some $k \times n$ matrix $U$ and some invertible symmetric $k \times k$ matrix $B$. Using the procedure outlined in the paragraphs following Lemma 2.9, we see that $G$ is a blowup of a graph $\tilde{G}$ corresponding to $\tilde{U}^{t} B \tilde{U}$, where $\tilde{U}$ and $B$ are defined as in the procedure. Lemma 2.9 then shows that $\tilde{G} \in \mathfrak{g}_{k}\left(\mathbb{F}_{q}\right)$.

Conversely, let $G$ be a blowup of some graph in $\left\{H \cup K_{1} \mid H \in \mathfrak{g}_{k}\left(\mathbb{F}_{q}\right)\right\}$ obtained by replacing each vertex $v_{i}$ of $H$ with a set of vertices $V_{i}$ and $K_{1}$ with any number of vertices. Deleting isolated vertices of $G$ does not change the minimum rank of $G$, so without loss of generality, we will assume that $G$ has no isolated vertices (which implies that $K_{1}$ was replaced with an empty set of vertices). Let $\overline{x_{1}}, \overline{x_{2}}, \ldots, \overline{x_{m}}$ be the projective equivalence classes of $\mathbb{F}_{q}^{k}-\overrightarrow{0}$, with each $x_{i}$ as a chosen representative from its class. Let $\tilde{U}=\left[\begin{array}{llll}x_{1} & x_{2} & \cdots & x_{m}\end{array}\right]$ and let $B$ be an invertible symmetric $k \times k$ matrix such that $\tilde{U}^{t} B \tilde{U}$ corresponds to the graph $H$. Form the matrix $\hat{U}=\left[\begin{array}{llll}X_{1} & X_{2} & \cdots & X_{m}\end{array}\right]$ by replacing each column $x_{i}$ of $\tilde{U}$ with the block $X_{i}$, where the columns of $X_{i}$ consist of $\left|V_{i}\right|$ copies of $x_{i}$. Then $\hat{U}^{t} B \hat{U}$ corresponds to $G$ and $\operatorname{rank} \hat{U}^{t} B \hat{U} \leq k$ since $B$ has rank $k$. Thus $\operatorname{mr}\left(\mathbb{F}_{q}, G\right) \leq k$, so $G \in \mathcal{G}_{k}\left(\mathbb{F}_{q}\right)$. $\square$

Now we will make this into a more explicit characterization of $\mathcal{G}_{k}\left(\mathbb{F}_{q}\right)$ by finding a suitable $\mathcal{B}$ for any $k$ and any $q$, thus enabling us to explicitly find $\mathfrak{g}_{k}\left(\mathbb{F}_{q}\right)$ for any $k$ and any $q$. 
3. Congruence classes of symmetric matrices over finite fields. Symmetric matrices represent symmetric bilinear forms and play an important role in projective geometry. Two congruent symmetric matrices represent the same symmetric bilinear form with respect to different bases. Because of their fundamental importance, congruence classes of symmetric matrices over finite fields have been studied and characterized for a long time in projective geometry. In this section, we have distilled the pertinent proofs of these characterizations from [1], [23], and [16] to give a suitable $\mathcal{B}$ for invertible symmetric $k \times k$ matrices over $\mathbb{F}_{q}$ for any $k$ and $q$. In the next section, we will expound more on the connection between the minimum rank problem and projective geometry.

We need the following elementary lemma.

Lemma 3.1. If a symmetric matrix $B=\left[\begin{array}{cc}C & D \\ D^{t} & E\end{array}\right]$, where $C$ is a square invertible matrix, then $B$ is congruent to $\left[\begin{array}{cc}C & O \\ O & E^{\prime}\end{array}\right]$, where $O$ is a zero matrix and $E^{\prime}$ is a square symmetric matrix of the same order as $E$.

Proof. Let $R=C^{-1} D$ so that $C R=D$. Then

$$
\begin{gathered}
\begin{array}{cc}
{\left[\begin{array}{cc}
I & O \\
-R^{t} & I
\end{array}\right]\left[\begin{array}{cc}
C & D \\
D^{t} & E
\end{array}\right]\left[\begin{array}{cc}
I & -R \\
O & I
\end{array}\right]} & =\left[\begin{array}{cc}
C & D \\
-R^{t} C+D^{t} & -R^{t} D+E
\end{array}\right]\left[\begin{array}{cc}
I & -R \\
O & I
\end{array}\right] \\
& =\left[\begin{array}{cc}
C & -C R+D \\
-R^{t} C+D^{t} & R^{t} C R-D^{t} R-R^{t} D+E
\end{array}\right] \\
& =\left[\begin{array}{cc}
C & O \\
O & E-D^{t} R
\end{array}\right],
\end{array} \\
\text { since }-C R+D=O=(-C R+D)^{t}=-R^{t} C+D^{t} \text {. }
\end{gathered}
$$

Lemma 3.2. Every symmetric matrix over $\mathbb{F}_{q}$ is congruent to a matrix of the form $\operatorname{diag}\left(a_{1}, a_{2}, \ldots, a_{s}, b_{1} H_{1}, b_{2} H_{2}, \ldots, b_{t} H_{t}\right)$, where $a_{i}, b_{i} \in \mathbb{F}_{q}, H_{i}=\left[\begin{array}{ll}0 & 1 \\ 1 & 0\end{array}\right]$, and $s$ and $t$ are nonnegative integers.

Proof. If $B$ is the zero matrix, then the result is true.

If $B$ is not the zero matrix, then the diagonal of $B$ has a nonzero entry or there is some $a_{i j} \neq 0, i \neq j$, so that $B$ has a principal submatrix of the form $\left[\begin{array}{cc}0 & a_{i j} \\ a_{i j} & 0\end{array}\right]=$ $a_{i j} H$, where $H=\left[\begin{array}{ll}0 & 1 \\ 1 & 0\end{array}\right]$.

In the first case, by using a suitable permutation, we may assume that $b_{11} \neq 0$. By Lemma $3.1, B$ is congruent to $\operatorname{diag}\left(b_{11}, B^{\prime}\right)$. 
In the second case, again by using a suitable permutation, we may assume that the upper left $2 \times 2$ principal submatrix is $a_{i j} H$. By Lemma 3.1, $B$ is congruent to $\operatorname{diag}\left(a_{i j} H, B^{\prime}\right)$.

Continue this process inductively with $B^{\prime}$. Then, again using a suitable permutation, $B$ is congruent to $\operatorname{diag}\left(a_{1}, a_{2}, \ldots, a_{s}, b_{1} H, b_{2} H, \ldots, b_{t} H\right)$.

We will now treat the even characteristic and odd characteristic cases separately.

3.1. Even characteristic. We first consider the case when $\mathbb{F}_{q}$ has even characteristic. First, we need a well-known result.

Lemma 3.3. Every element in a field of characteristic 2 is a square.

Corollary 3.4. Every symmetric matrix is congruent to $\operatorname{diag}\left(I_{s}, H_{1}, H_{2}, \ldots, H_{t}\right)$.

Proof. By Lemma 3.2, a symmetric matrix $A$ is congruent to a matrix

$$
B=\operatorname{diag}\left(a_{1}, a_{2}, \ldots, a_{s}, b_{1} H_{1}, b_{2} H_{2}, \ldots, b_{t} H_{t}\right) .
$$

Let

$$
C=\operatorname{diag}\left(\frac{1}{\sqrt{a_{1}}}, \frac{1}{\sqrt{a_{2}}}, \ldots, \frac{1}{\sqrt{a_{s}}}, \frac{1}{\sqrt{b_{1}}} I_{2}, \frac{1}{\sqrt{b_{2}}} I_{2}, \ldots, \frac{1}{\sqrt{b_{t}}} I_{2}\right) .
$$

Then $C^{t} B C=\operatorname{diag}\left(I_{s}, H_{1}, H_{2}, \ldots, H_{t}\right)$.

Let $B$ be a symmetric matrix in $\mathbb{F}_{q}$. Then according to Corollary $3.4, B$ is congruent to a matrix $C=\operatorname{diag}\left(I_{s}, H_{1}, H_{2}, \ldots, H_{t}\right)$, where each $H_{i}=\left[\begin{array}{ll}0 & 1 \\ 1 & 0\end{array}\right]$. Either $s=0$ or $s>0$. If $s>0$, then $\operatorname{diag}\left(I_{s}, H_{1}, H_{2}, \ldots, H_{t}\right)$, and thus $B$, is congruent to $I_{k}$. To see this, let

$$
A=\operatorname{diag}(1, H)=\left[\begin{array}{lll}
1 & 0 & 0 \\
0 & 0 & 1 \\
0 & 1 & 0
\end{array}\right] \quad \text { and } \quad C=\left[\begin{array}{lll}
1 & 1 & 1 \\
1 & 0 & 1 \\
0 & 1 & 1
\end{array}\right] .
$$

Then, since $\operatorname{char} \mathbb{F}_{q}=2$,

$$
C^{t}(A C)=\left[\begin{array}{lll}
1 & 1 & 0 \\
1 & 0 & 1 \\
1 & 1 & 1
\end{array}\right]\left[\begin{array}{lll}
1 & 1 & 1 \\
0 & 1 & 1 \\
1 & 0 & 1
\end{array}\right]=I_{3}
$$

If $s=0$, then $\operatorname{diag}\left(H_{1}, H_{2}, \ldots, H_{t}\right)$ and $B$ have even order and $B$ is congruent to $\operatorname{diag}\left(H_{1}, \ldots, H_{k / 2}\right)$.

The next lemma shows that these two cases are different. 
LEMMA 3.5. If a symmetric matrix $B$ has a zero diagonal, then every matrix congruent to $B$ has a zero diagonal.

Let $B$ be a symmetric matrix having a zero diagonal. If $v$ is the $k$ th column of a matrix $C$, then the $(k, k)$ entry of $C^{t} B C$ is $v^{t} B v$, which is zero, since

$$
v^{t} B v=\sum_{i, j} b_{i j} v_{i} v_{j}=\sum_{i} b_{i i} v_{i}^{2}+\sum_{i<j} b_{i j}\left(v_{i} v_{j}+v_{i} v_{j}\right)=\sum_{i} b_{i i} v_{i}^{2}=0 . \square
$$

The results in this subsection give us the following lemma.

Lemma 3.6. Let $q$ be even. To determine $\mathfrak{g}_{k}\left(\mathbb{F}_{q}\right)$, we may take $\mathcal{B}$ as follows: if $k$ is odd, then $\mathcal{B}=\left\{I_{k}\right\}$; if $k$ is even, then $\mathcal{B}=\left\{I_{k}, \operatorname{diag}\left(H_{1}, H_{2}, \ldots, H_{k / 2}\right)\right\}$, where $H_{i}=\left[\begin{array}{ll}0 & 1 \\ 1 & 0\end{array}\right]$.

3.2. Odd characteristic. We now consider the case when $\mathbb{F}_{q}$ has odd characteristic. We first need a well-known result.

Lemma 3.7. If $\mathbb{F}_{q}$ has odd characteristic and $\nu \in \mathbb{F}_{q}$, then there exists $c, d \in \mathbb{F}_{q}$ such that $c^{2}+d^{2}=\nu$.

Proof. Let $A=\left\{c^{2} \mid c \in \mathbb{F}_{q}\right\}$ and $B=\left\{\nu-d^{2} \mid d \in \mathbb{F}_{q}\right\}$. Since the map $\sigma: \mathbb{F}_{q}^{\times} \rightarrow$ $\mathbb{F}_{q}^{\times}$given by $\sigma: x \mapsto x^{2}$ has kernel $\{1,-1\}$, there are $(q-1) / 2$ squares in $\mathbb{F}_{q} \backslash\{0\}$. Including zero, there are then $(q+1) / 2$ squares in $\mathbb{F}_{q}$. Thus $|A|=|B|=(q+1) / 2$, so $A \cap B \neq \emptyset$, and $c^{2}=\nu-d^{2}$ for some $c, d \in \mathbb{F}_{q}$.

Since there are $(q-1) / 2$ nonzero squares in $\mathbb{F}_{q}$, given a nonsquare $\nu \in \mathbb{F}_{q}$, the set $\left\{\nu b^{2} \mid b \in \mathbb{F}_{q}, b \neq 0\right\}$ is a set of $(q-1) / 2$ nonsquares in $\mathbb{F}_{q}$. Consequently, every nonsquare is equal to $\nu b^{2}$ for some $b \in \mathbb{F}_{q}$.

The matrix $a H$ for any $a \in \mathbb{F}_{q}$ is congruent to a diagonal matrix:

$$
\left[\begin{array}{cc}
1 & 1 \\
-1 & 1
\end{array}\right]\left[\begin{array}{ll}
0 & a \\
a & 0
\end{array}\right]\left[\begin{array}{cc}
1 & -1 \\
1 & 1
\end{array}\right]=\left[\begin{array}{cc}
a & a \\
a & -a
\end{array}\right]\left[\begin{array}{cc}
1 & -1 \\
1 & 1
\end{array}\right]=\left[\begin{array}{cc}
2 a & 0 \\
0 & -2 a
\end{array}\right] .
$$

This fact combined with Lemma 3.2 shows that every symmetric matrix over $\mathbb{F}_{q}$ is congruent to a diagonal matrix.

LEMma 3.8. Every invertible symmetric $k \times k$ matrix $B$ over $\mathbb{F}_{q}$ is congruent to either $I_{k}$ or $\operatorname{diag}\left(I_{k-1}, \nu\right)$, where $\nu$ is any nonsquare in $\mathbb{F}_{q}$.

Let $C$ be an invertible diagonal matrix congruent to $B$, with $C=N^{t} B N$, and let $\nu$ be any nonsquare in $\mathbb{F}_{q}$.

By a permutation matrix $P$, let $D=P^{t} C P=\operatorname{diag}\left(b_{1}^{2}, b_{2}^{2}, \ldots, b_{s}^{2}, \nu c_{1}^{2}, \nu c_{2}^{2}, \ldots, \nu c_{t}^{2}\right)$, 
where the first $s$ elements of the diagonal of $D$ are squares in $\mathbb{F}_{q}$ and the last $t$ elements are nonsquares in $\mathbb{F}_{q}$.

Let $Q=\operatorname{diag}\left(b_{1}^{-1}, b_{2}^{-1}, \ldots, b_{s}^{-1}, c_{1}^{-1}, c_{2}^{-1}, \ldots, c_{t}^{-1}\right)$. Let $E=Q^{t} D Q=\operatorname{diag}\left(I_{s}, \nu I_{t}\right)$.

Let $c, d \in \mathbb{F}_{q}$ such that $c^{2}+d^{2}=\nu$. Let

$$
R=\nu^{-1}\left[\begin{array}{cc}
c & d \\
-d & c
\end{array}\right]
$$

Since $\operatorname{det} R=\nu^{-2}\left(c^{2}+d^{2}\right)=\nu^{-1} \neq 0, R$ is invertible. Note that

$$
R^{t}\left(\nu I_{2}\right) R=\nu R^{t} R=\nu \nu^{-2}\left(c^{2}+d^{2}\right) I_{2}=I_{2} .
$$

If $t$ is even, let $S=\operatorname{diag}\left(I_{s}, R_{1}, R_{2}, \ldots, R_{t / 2}\right)$, where $R_{i}=R$ for each $i$. Then $S^{t} E S=$ $I_{k}$. If $t$ is odd, let $S=\operatorname{diag}\left(I_{s}, R_{1}, R_{2}, \ldots, R_{(t-1) / 2}, 1\right)$. Then $S^{t} E S=\operatorname{diag}\left(I_{k-1}, \nu\right)$.

The next lemma shows that these two cases are in fact different and gives a simple criteria to determine which congruence class any symmetric matrix is in.

Lemma 3.9. If $\operatorname{det} B$ is a square (nonsquare) and $\hat{B}$ is congruent to $B$, then $\operatorname{det} \hat{B}$ is a square (nonsquare).

Proof. Let $\hat{B}=C^{t} B C$. Then $\operatorname{det} \hat{B}=(\operatorname{det} C)^{2}(\operatorname{det} B)$. Thus $\operatorname{det} B$ is a square if and only if $\operatorname{det} \hat{B}$ is a square.

Since $\operatorname{det} I_{k}=1$ is a square and $\operatorname{det}\left(\operatorname{diag}\left(I_{k-1}, \nu\right)\right)=\nu$ is a nonsquare, we can determine if a matrix is congruent to $I_{k}$ or congruent to $\operatorname{diag}\left(I_{k-1}, \nu\right)$ by whether the determinant is a square or not.

It appears then that $|\mathcal{B}|=2$. However, we can do better in one case since we only are concerned with whether an entry of $U^{t} B U$ is zero or nonzero and not with the actual value of the entry.

Definition 3.10. Let $B$ and $\hat{B}$ be matrices. If $\hat{B}=d C^{t} B C$ for some invertible matrix $C$ and some nonzero constant $d$, then $B$ and $\hat{B}$ are projectively congruent.

Since multiplying by a nonzero constant preserves the zero/nonzero pattern in a matrix over a field, if $B$ and $\hat{B}$ are projectively congruent, then $U^{t} B U$ and $U^{t} \hat{B} U$ give isomorphic graphs.

LEMMA 3.11. If $k$ is odd, then an invertible symmetric $k \times k$ matrix is projectively congruent to $I_{k}$.

Proof. Let $k=2 \ell-1$. We can see that $\operatorname{det}\left(\nu \operatorname{diag}\left(I_{k-1}, \nu\right)\right)=\nu^{2 \ell-1} \nu=\nu^{2 \ell}$ is a square. Thus $\operatorname{diag}\left(I_{k-1}, \nu\right)$ is projectively congruent to $I_{k}$.

The results in this subsection give us the following lemma. 


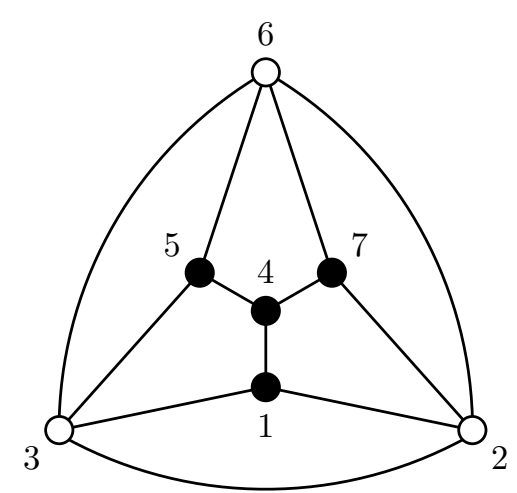

(a) $F 2 R 3$

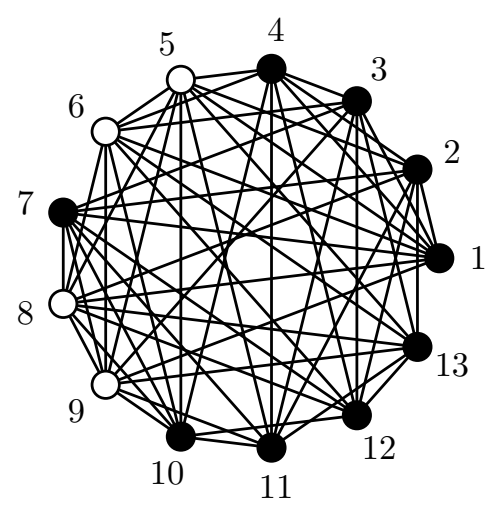

(b) $F 3 R 3$

Fig. 3.1: Graphs in Corollaries 3.14 and 3.15

Lemma 3.12. Let $q$ be odd. To determine $\mathfrak{g}_{k}\left(\mathbb{F}_{q}\right)$, we may take $\mathcal{B}$ as follows: if $k$ is odd, then $\mathcal{B}=\left\{I_{k}\right\}$; if $k$ is even, then $\mathcal{B}=\left\{I_{k}, \operatorname{diag}\left(I_{k-1}, \nu\right)\right\}$, where $\nu$ is any nonsquare in $\mathbb{F}_{q}$.

3.3. Summary. Combining Lemmas 3.6 and 3.12 , the results of this section can be summarized as the following theorem.

THEOREM 3.13. The set $\mathfrak{g}_{k}\left(\mathbb{F}_{q}\right)$ is the set of graphs of the matrices in $\left\{U^{t} B U \mid\right.$ $B \in \mathcal{B}\}$, where the columns of $U$ are a maximal set of nonzero vectors in $\mathbb{F}_{q}^{k}$ such that no vector is a multiple of another and $\mathcal{B}$ is given by:

1. if $k$ is odd, $\mathcal{B}=\left\{I_{k}\right\}$.

2. if $k$ is even and $\operatorname{char} \mathbb{F}_{q}=2, \mathcal{B}=\left\{I_{k}, \operatorname{diag}\left(H_{1}, H_{2}, \ldots, H_{k / 2}\right)\right\}$, where $H_{i}=$ $\left[\begin{array}{ll}0 & 1 \\ 1 & 0\end{array}\right]$.

3. if $k$ is even and $\operatorname{char} \mathbb{F}_{q} \neq 2, \mathcal{B}=\left\{I_{k}, \operatorname{diag}\left(I_{k-1}, \nu\right)\right\}$, where $\nu$ is any nonsquare in $\mathbb{F}_{q}$.

3.4. Examples of characterizations. As special cases of Theorem 3.13, we present the following corollaries which calculate $\mathfrak{g}_{k}\left(\mathbb{F}_{q}\right)$ for several $k$ and $q$. In the corollaries, we label a graph in $\mathfrak{g}_{k}\left(\mathbb{F}_{q}\right)$ using the pattern $F q R k$, signifying that it is a graph for the $\operatorname{mr}\left(\mathbb{F}_{q}, G\right) \leq k$ corollary. To compute these graphs, we used the software program Sage [32] and the Sage functions listed in Appendix A.

In these theorems, recall that $K_{1}$ does not have a loop.

Corollary 3.14. Let $G$ be any simple graph. Let F2R3 be the graph in Fig- 
ure 3.1(a). Then $\operatorname{mr}\left(\mathbb{F}_{2}, G\right) \leq 3$ (i.e., $G \in \mathcal{G}_{3}\left(\mathbb{F}_{2}\right)$ ) if and only if $G$ is a blowup graph of $F 2 R 3 \cup K_{1}$.

As matrices over $\mathbb{F}_{2}$, let

$$
U=\left[\begin{array}{lllllll}
0 & 1 & 0 & 1 & 0 & 1 & 1 \\
0 & 0 & 1 & 1 & 1 & 1 & 0 \\
1 & 1 & 1 & 1 & 0 & 0 & 0
\end{array}\right] \text { and } B=\left[\begin{array}{lll}
1 & 0 & 0 \\
0 & 1 & 0 \\
0 & 0 & 1
\end{array}\right]
$$

Then the graph F2R3 corresponds to the matrix

$$
U^{t} B U=\left[\begin{array}{lllllll}
1 & 1 & 1 & 1 & 0 & 0 & 0 \\
1 & 0 & 1 & 0 & 0 & 1 & 1 \\
1 & 1 & 0 & 0 & 1 & 1 & 0 \\
1 & 0 & 0 & 1 & 1 & 0 & 1 \\
0 & 0 & 1 & 1 & 1 & 1 & 0 \\
0 & 1 & 1 & 0 & 1 & 0 & 1 \\
0 & 1 & 0 & 1 & 0 & 1 & 1
\end{array}\right]
$$

Similarly, straightfoward matrix calculations give the following corollaries.

Corollary 3.15. Let $G$ be any simple graph. Let F3R3 be the graph in Figure 3.1(b). Then $\operatorname{mr}\left(\mathbb{F}_{3}, G\right) \leq 3$ (i.e., $G \in \mathcal{G}_{3}\left(\mathbb{F}_{3}\right)$ ) if and only if $G$ is a blowup graph of $F 3 R 3 \cup K_{1}$.

The next corollary gives the simplest previously-unknown result for which $\mathfrak{g}_{k}\left(\mathbb{F}_{q}\right)$ contains two graphs.

Corollary 3.16. Let $G$ be any simple graph. Let F2R4A and $F 2 R 4 B$ be the graphs in Figure 3.2. Then $\operatorname{mr}\left(\mathbb{F}_{2}, G\right) \leq 4$ (i.e., $G \in \mathcal{G}_{4}\left(\mathbb{F}_{2}\right)$ ) if and only if $G$ is a blowup graph of either $F 2 R 4 A \cup K_{1}$ or $F 2 R 4 B \cup K_{1}$.

4. Connection to projective geometry. As mentioned previously, the classifications of symmetric matrices in Section 3 are standard classification results in projective geometry. In this section, we first review appropriate terminology and highlight this connection to projective geometry. We will define slightly more terminology than is strictly necessary to help the reader see where these things fit into standard projective geometry. We then give some examples of how results in projective geometry can help us understand $\mathfrak{g}_{k}\left(\mathbb{F}_{q}\right)$ better. For further material, a definitive treatise on projective geometry is contained in the series [23] and [24].

4.1. Definitions and the connection. We start with basic definitions from projective geometry. 


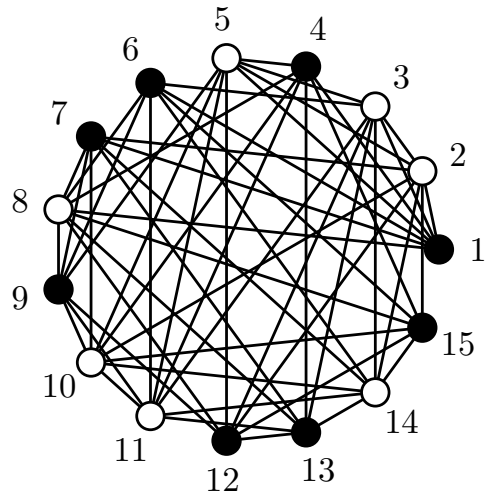

(a) $F 2 R 4 A$

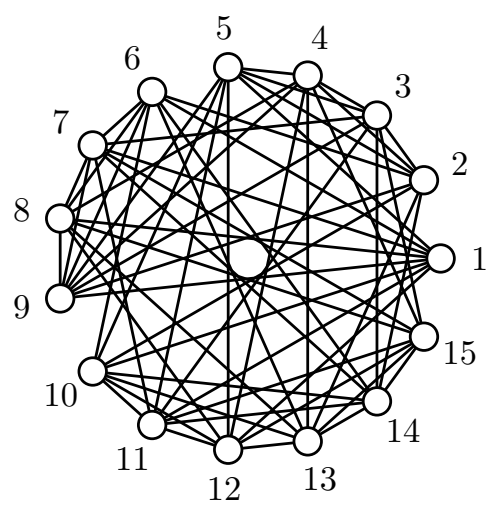

(b) $F 2 R 4 B$

Fig. 3.2: Graphs in Corollary 3.16

Definition 4.1. Let $V=\mathbb{F}_{q}^{n+1}$, the vector space of dimension $n+1$ over $\mathbb{F}_{q}$. For $x, y \in V-\overrightarrow{0}$, we define an equivalence relation by

$$
x \sim y \Longleftrightarrow x=c y, \quad \text { where } c \in \mathbb{F}_{q} \text { and } c \neq 0 .
$$

Denote the equivalence class containing $x \in V-\overrightarrow{0}$ as $\bar{x}=\left\{c x \mid c \in \mathbb{F}_{q}\right.$ and $\left.c \neq 0\right\}$. Geometrically, we can think of the class $\bar{x}$ as the set of non-origin points on a line passing through $x$ and the origin in $V$. These equivalence classes form the projective geometry $P G(n, q)$ of (projective) dimension $n$ and order $q$. The equivalence classes are called the points of $P G(n, q)$. Each subspace of dimension $m+1$ in $V$ corresponds to a subspace of (projective) dimension $m$ in $P G(n, q)$. If a projective geometry has (projective) dimension 2 , then it is called a projective plane.

Note that there is a shift by one in dimension between a vector space $V$ and its subspaces and the projective geometry associated with $V$ and its subspaces. To help the reader, we will use the nonstandard term projective dimension (or "pdim") when dealing with the dimension of a projective geometry.

Definition 4.2. Let $\mathcal{S}$ be the set of subspaces of $P G(n, q)$. A correlation $\sigma: \mathcal{S} \rightarrow \mathcal{S}$ is a bijective map such that for any subspaces $R, T \in \mathcal{S}, R \subseteq T$ implies that $\sigma(T) \subseteq \sigma(R)$ and $\operatorname{pdim} \sigma(R)=n-1-\operatorname{pdim} R$. A polarity is a correlation $\sigma$ of order 2 (i.e., $\sigma^{2}=1$, the identity map).

Note that any polarity $\sigma$ maps points in $\mathcal{S}$ to hyperplanes (subspaces of projective dimension $n-1$ in $\mathcal{S}$ ) and hyperplanes to points. Since $\sigma^{2}=1$, we have $Y=\sigma(\bar{x})$ if and only if $\sigma(Y)=\bar{x}$, so $\sigma$ induces a bijection between points and hyperplanes. This bijection leads to the next definition. 
Definition 4.3. Let $\sigma$ be a polarity on $P G(n, q)$. Let $\bar{x}, \bar{y}$ be points in $P G(n, q)$. We say that $\sigma(\bar{x})$ is the polar (hyperplane) of $\bar{x}$ and $\bar{x}$ is the pole of $\sigma(\bar{x})$. If $\bar{y} \in \sigma(\bar{x})$, then $\bar{x} \in \sigma(\bar{y})$ and we say that $\bar{x}$ and $\bar{y}$ are conjugate points. If $\bar{x} \in \sigma(\bar{x})$, then we say that $\bar{x}$ is self-conjugate or absolute. Similarly, if $S$ is a subspace of $P G(n, q)$, then $S$ is absolute if $\sigma(S) \subseteq S$ or $S \subseteq \sigma(S)$. A subspace of $P G(n, q)$ consisting of absolute points is called isotropic.

The next definition gives the connection with symmetric matrices.

Definition 4.4. Let $B$ be an $(n+1) \times(n+1)$ invertible symmetric matrix over $\mathbb{F}_{q}$. Define $\sigma: \mathcal{S} \rightarrow \mathcal{S}$ by $\sigma: R \mapsto R^{\perp}$, where the orthogonality relation is defined by the nondegenerate symmetric bilinear form represented by $B$ (i.e., $R^{\perp}=\left\{\bar{y} \mid x^{t} B y=\right.$ Oforall $\bar{x} \in R\}$ ). We call $\sigma$ the polarity associated with $B$.

The fact that the $\sigma$ in the previous definition is a polarity is easy to check.

Let $M_{1}$ and $M_{2}$ be symmetric matrices. Let $\sigma_{1}$ and $\sigma_{2}$ be the associated polarities, respectively. Two polarities are equivalent if the matrices are projectively congruent, i.e., $\sigma_{1}$ is equivalent to $\sigma_{2}$ if $M_{1}=d C^{t} M_{2} C$ for some nonzero $d$ and invertible matrix $C$. Thus there is a unique polarity associated with each matrix given in Theorem 3.13.

We now summarize from [23, Section 2.1.5] the classification of polarities that are associated with symmetric matrices. Let $B$ be an invertible symmetric matrix over $\mathbb{F}_{q}$. Let $\sigma$ be the polarity associated with $B$.

- If $q$ is odd, then $\sigma$ is called an ordinary polarity.

If $B$ has even order, then the associated polarity is either a hyperbolic polarity or an elliptic polarity. The correspondence between these types of polarities and the matrices in $\mathcal{B}$ from Theorem $3.13(3)$ is slightly nontrivial and is summarized in [23, Corollary 5.19].

If $B$ has odd order, then $\sigma$ is a parabolic polarity, which corresponds to $\mathcal{B}$ in Theorem 3.13(1).

- If $q$ is even and $b_{i i}=0$ for all $i$, then $\sigma$ is a null polarity (or in alternate terminology, $\sigma$ is a symplectic polarity). Note that this only occurs when $B$ has even order since otherwise $B$ is not invertible. This case corresponds to the non-identity matrix in the $\mathcal{B}$ in Theorem 3.13(2).

- If $q$ is even and there is some $b_{i i} \neq 0$, then $\sigma$ is a pseudo-polarity. This case corresponds to the identity matrix in $\mathcal{B}$ in Theorem 3.13(1) or (2).

We pause to note that there are polarities that are not associated with symmetric matrices. However, since we are only concerned about symmetric matrices, we will restrict ourselves to this case. Information about polarities not associated with symmetric matrices may also be found in [23]. 
We now examine the connection to graphs by recalling the definition of a polarity graph.

Definition 4.5. Let $B$ be an invertible symmetric $(n+1) \times(n+1)$ matrix over $\mathbb{F}_{q}$ and let $\sigma$ be the associated polarity. The polarity graph of $\sigma$ has as its vertices the points of $P G(n, q)$ and as its edges $\left\{\bar{x} \bar{y} \mid x^{t} B y=0\right\}$. In a polarity graph, $\bar{x}$ is adjacent to $\bar{y}$ exactly when $\bar{x}$ and $\bar{y}$ are conjugate (i.e., $x$ and $y$ are orthogonal with respect to $B$ ). In standard literature, loops are not allowed in polarity graphs. However, for our purposes, loops convey needed information, so a vertex $\bar{x}$ in a polarity graph has a loop if and only if $\bar{x}$ is absolute (i.e., $x^{t} B x=0$, where $B$ is an invertible symmetric matrix associated with the polarity).

In Theorem 3.13, the vertices of a graph in $\mathfrak{g}_{k}\left(\mathbb{F}_{q}\right)$ represent the points of the projective geometry $P G(k-1, q)$ and an edge is drawn if the corresponding points are not conjugate (i.e., $x^{t} B y \neq 0$ ). Thus, the graphs in Theorem 3.13 are exactly the complements of polarity graphs. Recall that when dealing with looped graphs, a vertex is looped in the complement of a graph if and only if it is nonlooped in the original graph.

Using this connection, we can restate Theorem 3.13:

THEOREM 4.6. The set $\mathfrak{g}_{k}\left(\mathbb{F}_{q}\right)$ is the set of complements of the (looped) polarity graphs of the polarities on $P G(k-1, q)$ that are associated with symmetric matrices.

4.2. Consequences of the connection. With the main theorem stated as in Theorem 4.6, we can use a variety of known results about polarity graphs to derive results about graphs in $\mathfrak{g}_{k}\left(\mathbb{F}_{q}\right)$. In this section, we list a few consequences of Theorem 4.6.

An elementary result in projective geometry gives us the size of the graphs in $\mathfrak{g}_{k}\left(\mathbb{F}_{q}\right)$. While this result could have been realized from the statement in Theorem 3.13, it also naturally follows as a consequence of Theorem 4.6.

THEOREM 4.7. Every graph in $\mathfrak{g}_{k}\left(\mathbb{F}_{q}\right)$ has $\frac{q^{k}-1}{q-1}$ vertices.

Proof. There are $q^{k}-1$ vectors in $\mathbb{F}_{q}^{k}-\overrightarrow{0}$. Since there are $q-1$ nonzero constants in $\mathbb{F}_{q}$, there are $q-1$ elements in each equivalence class in $P G(k-1, q)$, so there are $\frac{q^{k}-1}{q-1}$ points in $P G(k-1, q)$.

The following observation follows directly from Theorem 4.6 and restates the criteria for an edge in a graph in $\mathfrak{g}_{k}\left(\mathbb{F}_{q}\right)$ in several ways.

ObSeRvation 4.8. Let $G \in \mathfrak{g}_{k}\left(\mathbb{F}_{q}\right)$ and let $u$ and $v$ be (not necessarily distinct) vertices in $G$. Let $\sigma$ be the polarity corresponding to $G$ and let $B$ be an invertible symmetric matrix corresponding to $\sigma$. Then $u v$ is an edge in $G$ if and only if: 
1. $u^{t} B v \neq 0$ (equivalently, $v^{t} B u \neq 0$ ), or equivalently,

2. $u$ and $v$ are not conjugate points, or equivalently,

3. $u \notin \sigma(v)$ (equivalently, $v \notin \sigma(u)$ ).

Corollary 4.9. A graph $G \in \mathfrak{g}_{k}\left(\mathbb{F}_{q}\right)$ is regular of degree $q^{k-1}$ (using the convention that a loop adds one to the degree of a vertex). Let $v \in G$ and let $\sigma$ be the polarity associated with $G$. Since the hyperplane $\sigma(v)$ contains $\frac{q^{k-1}-1}{q-1}$ points, this is the degree of a $v$ in the complement of $G$. Thus the degree of $v$ in $G$ is

$$
\frac{q^{k}-1}{q-1}-\frac{q^{k-1}-1}{q-1}=q^{k-1}
$$

In light of Observation 4.8, determining the numbers of looped and nonlooped vertices in $G$ is equivalent to finding the numbers of absolute points of the polarities of $P G(k-1, q)$.

TheOrem 4.10. Let $\mathbb{F}_{q}$ be a finite field having characteristic 2. One graph in $\mathfrak{g}_{k}\left(\mathbb{F}_{q}\right)$ will have $\frac{q^{k-1}-1}{q-1}$ nonlooped vertices. If $k$ is even, then the additional graph in $\mathfrak{g}_{k}\left(\mathbb{F}_{q}\right)$ will have all nonlooped vertices.

Proof. In a field of characteristic 2, since

$$
x^{t} B x=\sum_{i, j} b_{i j} x_{i} x_{j}=\sum_{i} b_{i i} x_{i}^{2}+\sum_{i<j} b_{i j}\left(x_{i} x_{j}+x_{i} x_{j}\right)=\sum_{i} b_{i i} x_{i}^{2}=\left(\sum_{i} \sqrt{b_{i i}} x_{i}\right)^{2},
$$

a point $\bar{x}$ is absolute if and only if $\sum_{i} \sqrt{b_{i i}} x_{i}=0$.

In a pseudo-polarity, the set of absolute points is the hyperplane $\sum_{i} \sqrt{b_{i i}} x_{i}=0$. Since a hyperplane of $P G(k-1, q)$ is a projective geometry of projective dimension $k-2$, there are $\frac{q^{k-1}-1}{q-1}$ nonlooped vertices in this graph.

In a null polarity, $b_{i i}=0$ for all $i$. Therefore every vertex is nonlooped (i.e., there are $\frac{q^{k}-1}{q-1}$ nonlooped vertices). A null polarity occurs when $k$ is even. $\square$

For the odd characteristic case, we will directly apply a standard result in projective geometry about the number of absolute points in ordinary polarities.

Theorem 4.11 ([24, Theorem 22.5.1(b)]). Let $q$ be odd. Then the number of absolute points in a polarity in $P G(k-1, q)$ is given by:

- $\frac{\left(q^{m}-1\right)\left(q^{m-1}+1\right)}{q-1}$ or $\frac{\left(q^{m}+1\right)\left(q^{m-1}-1\right)}{q-1}$ if $k=2 m$ is even;

- $\frac{q^{2 m}-1}{q-1}$ if $k=2 m+1$ is odd.

Corollary 4.12. Let $q$ be odd. If $k=2 m$ is even, then the two graphs in $\mathfrak{g}_{k}\left(\mathbb{F}_{q}\right)$ will have $\frac{\left(q^{m}-1\right)\left(q^{m-1}+1\right)}{q-1}$ and $\frac{\left(q^{m}+1\right)\left(q^{m-1}-1\right)}{q-1}$ nonlooped vertices, respectively. If $k=2 m+1$ is odd, then the graph in $\mathfrak{g}_{k}\left(\mathbb{F}_{q}\right)$ will have $\frac{q^{2 m}-1}{q-1}$ nonlooped vertices. 
We conclude by applying a few standard results for polarities over $P G(2, q)$ (a projective plane) to give results about $\mathfrak{g}_{3}\left(\mathbb{F}_{q}\right)$ and the minimum rank problem. We note that the polarity graphs of $P G(2, q)$ for any $q$ are the Erdős-Rényi graphs from extremal graph theory (see [19], [20], or [12]). For a survey of interesting properties of the Erdős-Rényi graphs and their subgraphs, see [30] or [34, Chapter 3].

Theorem 4.13. If $G \in \mathfrak{g}_{3}\left(\mathbb{F}_{q}\right)$, then the nonlooped vertices in $G$ form a clique.

Proof. Suppose that $u$ and $v$ are distinct nonadjacent nonlooped vertices in $G$. Then $u$ and $v$ are absolute vertices and $u \in \sigma(u) \cap \sigma(v)$ and $v \in \sigma(u) \cap \sigma(v)$. This is a contradiction since the intersection of any two distinct lines in $P G(2, q)$ is a single point.

If $G \in \mathfrak{g}_{3}\left(\mathbb{F}_{q}\right)$, the formulas in Theorem 4.10 and Corollary 4.12 imply that $G$ has $q+1$ nonlooped vertices. This combined with Corollary 4.9 and Theorem 4.13 gives the following corollary.

COROLlaRY 4.14. If $G \in \mathfrak{g}_{3}\left(\mathbb{F}_{q}\right)$, then each nonlooped vertex is adjacent to $q$ nonlooped vertices and $q^{2}-q$ looped vertices.

Theorem 4.13 also gives us the following theorem.

THEOREM 4.15. Let $G=K_{s_{1}, s_{2}, \ldots, s_{n}}$, a simple complete multipartite graph. If $q \geq n-1$, then $\operatorname{mr}\left(\mathbb{F}_{q}, G\right) \leq 3$.

Proof. Let $G=K_{s_{1}, s_{2}, \ldots, s_{n}}$. Then $G$ is a blowup graph of $K_{n}$, where each vertex of $K_{n}$ is nonlooped. Since the graph in $\mathfrak{g}_{3}\left(\mathbb{F}_{q}\right)$ contains a clique of $q+1$ nonlooped vertices, if $q+1 \geq n$, then $G$ is a blowup graph of the graph in $\mathfrak{g}_{3}\left(\mathbb{F}_{q}\right)$.

We can now construct an interesting family of simple graphs.

THEOREM 4.16. For every integer $n \geq 1$, let $G_{n}$ be a simple complete multipartite graph $H_{1} \vee H_{2} \vee \cdots \vee H_{n}$ where each $H_{i}$ is an independent set with $s_{i}>(n-1)^{2}$ vertices. We then have $\operatorname{mr}\left(\mathbb{F}_{q}, G_{n}\right) \leq 3$ if and only if $q \geq n-1$.

Proof. If $q \geq n-1$, then $\operatorname{mr}\left(\mathbb{F}_{q}, G_{n}\right) \leq 3$ by Theorem 4.15 .

Conversely, let $q<n-1$. Let $I$ be the graph in $\mathfrak{g}_{3}\left(\mathbb{F}_{q}\right)$ and let $I_{1}$ and $I_{2}$ be the subgraphs of $I$ induced by the looped and nonlooped vertices of $I$, respectively. Since $I_{1}$ has $q^{2}$ vertices, any blowup of $I_{1}$ containing more than $q^{2}$ vertices will contain an edge by the pigeon-hole principle. Since the vertices in each $H_{i}$ form an independent set of size $s_{i}>(n-1)^{2}>q^{2}$, at least one vertex in each $H_{i}$ must be a blowup of a vertex in $I_{2}$. Furthermore, since the vertices of each $H_{i}$ have the same neighbors, we can assume without loss of generality that all of the vertices of each $H_{i}$ are blowups of vertices of $I_{1}$. Thus $G_{n}$ is a blowup of $I_{2}$. However, any blowup of $I_{2}$ will be of the form $K_{t_{1}, t_{2}, \ldots, t_{q+1}}$ since $I$ has $q+1$ nonlooped vertices, but $G_{n}$ is not of this form 
since $q+1<n$.

5. Conclusion. We have suceeded in classifying the structure of graphs in $\mathcal{G}_{k}\left(\mathbb{F}_{q}\right)$ for any $k$ and any $q$. We have also shown how this classification relates to projective geometry. We have applied a few results of projective geometry to give results in the minimum rank problem.

We conclude with a short list of open questions and topics for further investigation. First, there are many results about polarity graphs that could potentially yield results for the minimum rank problem. What other facts from projective geometry can be applied to give results in the minimum rank problem over finite fields?

The structural characterization in this paper gives rise to a theoretical procedure for determining the minimum rank of any graph over a finite field. How can this procedure be efficiently implemented? How can the results of Ding and Kotlov [18] be combined with the classification in this paper to yield results on minimal forbidden subgraphs describing $\mathcal{G}_{k}\left(\mathbb{F}_{q}\right)$ ? The author has implemented such an algorithm and has some preliminary results on the numbers of forbidden subgraphs describing $\mathcal{G}_{k}\left(\mathbb{F}_{q}\right)$ for different values of $k$ and $q$.

Finally, there is still ongoing research investigating the structure of polarity graphs. For example, Jason Williford [34], Michael Newman, and Chris Godsil [21] have recently investigated the sizes of independent sets in polarity graphs. Are there results in the minimum rank problem that would aid in answering questions about the structure of polarity graphs?

Acknowledgment. The author thanks Wayne Barrett and Don March for work in the early part of this research, including the proof of Theorem 2.2 and early computational experiments, as well as Willem Haemers for pointing out that the graph $F 2 R 3$ is related to the Fano projective plane, which led to the investigation of the connection to projective geometry. Most of this research was done for the author's Ph.D. dissertation and the support of Brigham Young University is gratefully acknowledged. Additionally, the support of Iowa State University during the editing phase is gratefully acknowledged.

\section{REFERENCES}

[1] A. Adrian Albert. Symmetric and alternate matrices in an arbitrary field. I. Trans. Amer. Math. Soc., 43(3):386-436, 1938.

[2] Marina Arav, Frank J. Hall, Selcuk Koyuncu, Zhongshan Li, and Bhaskara Rao. Rational realizations of the minimum rank of a sign pattern matrix. Linear Algebra Appl., 409:111125, 2005. 
[3] Efrat Bank. Symmetric matrices with a given graph. Master's thesis, Technion-Israel Institute of Technology, February 2007.

[4] Francesco Barioli and Shaun Fallat. On the minimum rank of the join of graphs and decomposable graphs. Linear Algebra Appl., 421(2-3):252-263, 2007.

[5] Francesco Barioli, Shaun Fallat, and Leslie Hogben. Computation of minimal rank and path cover number for certain graphs. Linear Algebra Appl., 392:289-303, 2004.

[6] Francesco Barioli, Shaun Fallat, and Leslie Hogben. On the difference between the maximum multiplicity and path cover number for tree-like graphs. Linear Algebra Appl., 409:13-31, 2005.

[7] Francesco Barioli, Shaun Fallat, and Leslie Hogben. A variant on the graph parameters of Colin de Verdière: implications to the minimum rank of graphs. Electron. J. Linear Algebra, 13:387-404 (electronic), 2005.

[8] Wayne Barrett, Jason Grout, and Raphael Loewy. The minimum rank problem over the finite field of order 2: Minimum rank 3. Linear Algebra and its Applications, 430(4):890 - 923, 2009.

[9] Wayne Barrett, Hein van der Holst, and Raphael Loewy. Graphs whose minimal rank is two. Electron. J. Linear Algebra, 11:258-280 (electronic), 2004.

[10] Wayne Barrett, Hein van der Holst, and Raphael Loewy. Graphs whose minimal rank is two: the finite fields case. Electron. J. Linear Algebra, 14:32-42 (electronic), 2005.

[11] Américo Bento and António Leal Duarte. On Fiedler's characterization of tridiagonal matrices over arbitrary fields. Linear Algebra Appl., 401:467-481, 2005.

[12] W. G. Brown. On graphs that do not contain a Thomsen graph. Canad. Math. Bull., 9:281-285, 1966.

[13] Guantao Chen, Frank J. Hall, Zhongshan Li, and Bing Wei. On ranks of matrices associated with trees. Graphs Combin., 19(3):323-334, 2003.

[14] Nathan L. Chenette, Sean V. Droms, Leslie Hogben, Rana Mikkelson, and Olga Pryporova. Minimum rank of a tree over an arbitrary field. Electron. J. Linear Algebra, 16:183-186 (electronic), 2007.

[15] Gordon Royle Chris Godsil. Algebraic Graph Theory. Springer-Verlag, 2001.

[16] P. M. Cohn. Basic algebra. Springer-Verlag London Ltd., London, 2003. Groups, rings and fields.

[17] Yves Colin de Verdière. Multiplicities of eigenvalues and tree-width of graphs. J. Combin. Theory Ser. B, 74(2):121-146, 1998.

[18] Guoli Ding and Andreı̆ Kotlov. On minimal rank over finite fields. Electron. J. Linear Algebra, 15:210-214 (electronic), 2006.

[19] P. Erdős and A. Rényi. On a problem in the theory of graphs. Magyar Tud. Akad. Mat. Kutató Int. Közl., 7:623-641 (1963), 1962.

[20] P. Erdős, A. Rényi, and V. T. Sós. On a problem of graph theory. Studia Sci. Math. Hungar., 1:215-235, 1966.

[21] C D Godsil and M W Newman. Eigenvalue bounds for independent sets, 2005.

[22] Frank J. Hall, Zhongshan Li, and Bhaskara Rao. From Boolean to sign pattern matrices. Linear Algebra Appl., 393:233-251, 2004.

[23] J. W. P. Hirschfeld. Projective geometries over finite fields. Oxford Mathematical Monographs. The Clarendon Press Oxford University Press, New York, second edition, 1998.

[24] J. W. P. Hirschfeld and J. A. Thas. General Galois geometries. Oxford Mathematical Monographs. The Clarendon Press Oxford University Press, New York, 1991. , Oxford Science Publications.

[25] Liang-Yu Hsieh. On Minimum Rank Matrices having a Prescribed Graph. PhD thesis, University of Wisconsin, Madison, 2001.

[26] C. R. Johnson and António Leal Duarte. The maximum multiplicity of an eigenvalue in a matrix whose graph is a tree. Linear and Multilinear Algebra, 46(1-2):139-144, 1999. Invariant 
factors.

[27] Charles R. Johnson and Carlos M. Saiago. Estimation of the maximum multiplicity of an eigenvalue in terms of the vertex degrees of the graph of a matrix. Electron. J. Linear Algebra, 9:27-31 (electronic), 2002.

[28] János Komlós, Gábor N. Sárközy, and Endre Szemerédi. Blow-up lemma. Combinatorica, 17(1):109-123, 1997.

[29] Peter M. Nylen. Minimum-rank matrices with prescribed graph. Linear Algebra Appl., 248:303316, 1996.

[30] T. D. Parsons. Graphs from projective planes. Aequationes Math., 14(1-2):167-189, 1976.

[31] John Sinkovic. The relationship between the minimal rank of a tree and the rank-spreads of the vertices and edges. Master's thesis, Brigham Young University, December 2006.

[32] William Stein. Sage Mathematics Software (Version 2.8.15). The Sage Group, 2007. http: //www.sagemath.org.

[33] Hein van der Holst. Graphs whose positive semi-definite matrices have nullity at most two. Linear Algebra Appl., 375:1-11, 2003.

[34] Jason Williford. Constructions in finite geometry with applications to graphs. PhD thesis, University of Delaware, 2004.

\section{Appendix A. Sage code to generate graphs.}

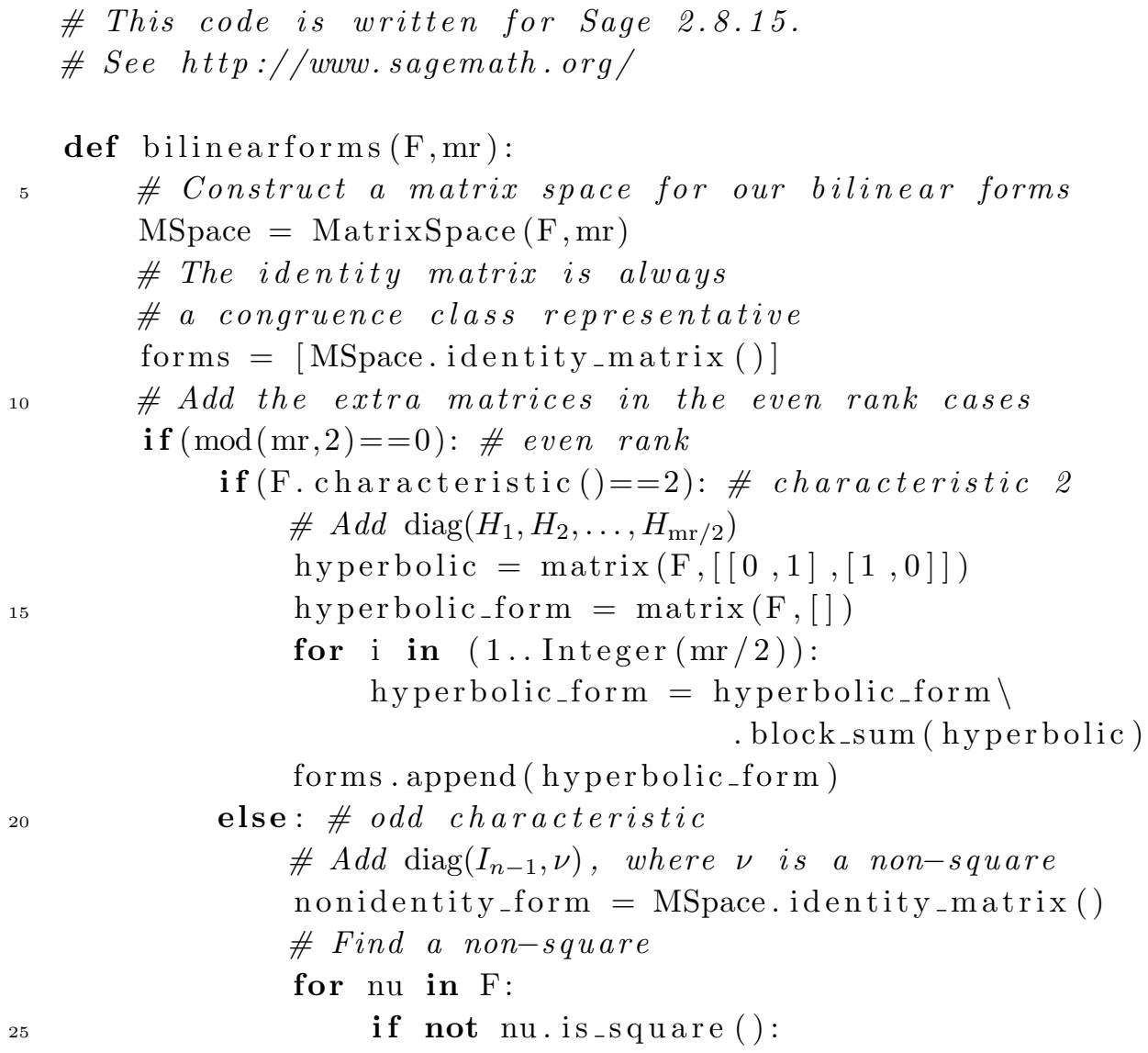




\section{break}

if $\mathrm{nu}$. is _ square ( ):

raise NotImplementedError, ।

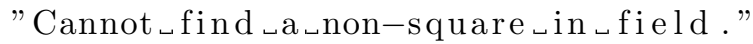

return forms

def get_matrices (F, mr):

return graphs

def get_graphs (F, mr):

$\mathrm{U}, \mathrm{B}=$ get_matrices $(\mathrm{F}, \mathrm{mr})$ product_matrices $=[\mathrm{U} . \operatorname{transpose}() * \mathrm{~b} * \mathrm{U}$ for $\mathrm{b}$ in $\mathrm{B}]$ graphs $=[$ Graph $(\mathrm{m})$ for $\mathrm{m}$ in product_matrices $]$

for $i$ in range(len(graphs)): graphs [i]. loops (true); graphs $[i]$.add_edges $([[j, j]$ for $j$ in range(len (graphs[i])) । if product_matrices $[\mathrm{i}][\mathrm{j}, \mathrm{j}]$ $!=0]$ )

def show_graphs $(\mathrm{F}, \mathrm{mr})$ :

for $\mathrm{g}$ in get_graphs $(\mathrm{F}, \mathrm{mr})$ : \# Vertices with loops are black, others are white vcolors $=\{$ 'black': g. loop_vertices ()$, \backslash$ 'white': [ $\mathrm{i}$ for $\mathrm{i}$ in g.vertices () if i not in g.loop_vertices ()]\} g. $\operatorname{show}($ layout=' circular', vertex_colors=vcolors , । vertex_labels=false )

\# To retrieve the matrices for graphs in $\mathfrak{g}_{3}\left(\mathbb{F}_{2}\right)$ : 
Electronic Journal of Linear Algebra ISSN 1081-3810

A publication of the International Linear Algebra Society

ELA

\# To retrieve the graphs in $\mathfrak{g}_{3}\left(\mathbb{F}_{2}\right)$ :

graph_list $=$ get_graphs $(\mathrm{F}=$ FiniteField $(2), \operatorname{mr}=3)$

\# To display the graphs with vertices colored appropriately: show_graphs $(\mathrm{F}=\mathrm{F}$ initeField $(2), \mathrm{mr}=3)$ 